\title{
Resource Information Retrieval Method Based on Name Service in Intelligent Agriculture
}

\author{
Xiaolong Luo ${ }^{1, a}$, Ye Tian ${ }^{2, b}$, Anping Xiong ${ }^{1, c}$ \\ 1 Chongqing University of Posts and Telecommunications School of computer science and \\ technology, Chongqing, China \\ 2 Computer Network Information Center, Chinese Academy of Sciences, Beijing China \\ ax|910128@qq.com, btianye@niot.cn, ${ }^{\mathrm{a}}$ xiongap@cqupt.edu.cn
}

\begin{abstract}
Keywords: Intelligent agriculture; retrieval service; associated resources discovery; expansibility. Abstract. Intelligent agriculture is the inevitable development trend of modern agriculture. Sharing the sensing data of agricultural production activities on internet to make it to be found is the premise of agricultural products supervision and agricultural decision. However, the relationship between agricultural products and perception data is complex, it is unable to obtain all the associated information with crawler, and hence the existing retrieval schemes cannot effectively solve the query demand of associated data resources of agricultural products. This paper puts forward a resource data retrieval method based on naming service, which makes clearly the association between products by identification. Finally, this paper carries out evaluation towards query efficiency, and verifies that the scheme has a good expansibility in heterogeneous network environment.
\end{abstract}

\section{Introduction}

Intelligent agriculture is one of the important directions in the application and development of Internet of Things (IOT) [1]. As the advanced stage of agricultural production, it can bring inestimable impetus and unlimited prospects. There are different definitions of intelligent agriculture [2], but the essences are the application of IOT in agriculture, including the sensing, monitor, treatment of various kinds of equipment on agricultural production, so as to provide a variety of valuable data services for agricultural producers and consumers. All kinds of data service support are various sensing data. Through these data, consumers can get more information on agricultural products, national regulatory authorities can regulate agricultural production, and agricultural service providers can provide accurate production suggestions for farmers. In order to better apply the sensing data, they need to be shared on the internet to be retrieved.

However, the sensing data in intelligent agriculture has characteristics of huge scale, temporal-spatial correlation, high redundancy and multi scalar, which determines the many challenges faced in retrieving data resources [3]. The resources aimed by traditional Internet retrieval are mainly web data based on static document [4], once the data releases, the content rarely changes[5], so the internet search engine can get the page keyword by crawler, establish inverted index to implement resource search. Most resources in intelligent agricultural are objects abstracted from the real world and sensing data information on real-time describing objects. Due to the complex relationship between objects [6], the sensing information of one object can also describe other objects associated with it, but these implied relationship cannot be fully obtained by crawler and thus establishing the index. Therefore, the traditional Internet search methods cannot effectively solve the query needs of all its related sensing data through object retrieval. What's more, the data is huge in intelligent agricultural and share data by opening the query interface, while users want to get different data service in the network provided by different applications through a unified query service. Therefore, what the IOT retrieval faces is a large-scale heterogeneous network environment, which requires a strong expansibility to the retrieval system.

In order to solve the above problems, this paper carries out the study on IOT resources retrieval scheme. It expresses the relationship between objects by using the identification method, maintain the association in real world with distributed index tree method. On this basis, this paper designs the 
retrieval method to enable it to get all the data resources related to query objects in heterogeneous network environment. Finally, it evaluates the query efficiency and verifies that this scheme has a good expansibility in heterogeneous network environment.

\section{Related Research}

The academic circle has been paid attention to the search of resource objects under the IOT environment, many researches have been done on the IOT retrieval technique based on many different starting points, and some achievements have been achieved.

Snoogle [7] system deploys sensor on real entity to keep the text description of a set of keywords related to the entity. The index points save the sensors within the administration scope, all index points are maintained unified by key index points. Query request is sent to index points or key index points according to the area, and returns the best results according to keyword matching. Such a centralized management makes the Snoogle system unable to be applied to large-scale heterogeneous network environment, its extendibility is not high.

MAX [8] system distributes the RFID with keywords for physical entity and deploys it in substation. For the base station, it logically expresses a static and immovable place, and is responsible for managing the sub stations within the scope. The top MAX server manages and maintains the catalog of all base stations and the location information. When searching, the server broadcasts layer by layer to get the label list matched with the keyword. Although it can reduce resources consumption in the index maintenance, it seriously increases the communication expenses, so it is also not applicable to large-scale heterogeneous network environment.

The core idea of WoT [9] is to share the resources and services of physical entity in the form of a web page by virtue of infrastructure resources of the Internet, thus to transfer the IOT resource retrieval service to the Internet search process. Dyser [10] system is a kind of real-time IOT search engine based on the idea of WoT.

Dyser retrieval system applies existing network infrastructure to release the service resource of sensor and physical entity in the form of Web pages, and create the retrieval of physical entity, so as to make users quickly search the physical entity that fits a specified state. The system proposes to realize the dynamic attribute search by establishing an entity prediction model by virtue of historical data information. Dyser is a flexible, extensible and universal search service. However, this system needs to determine the relationship between physical entities and sensors in advance, meanwhile, the sensors cannot automatically establish relations with all relevant entities, thus making users unable to acquire all resources of sensing data associated with the objects.

All relevant researches above have solved the problem to search for specified entity object on the internet in a certain extent, but for some practical applications, especially for the conditions of huge resource data scale, heterogeneous and association between objects in intelligent agriculture, they still can't meet the query requirements to retrieve all sensing data related to the object in specific realization. Therefore, based on the above schemes, this paper studies a retrieval method on the basis of name service to solve above requirements.

\section{Resource Retrieval Method Based on Name Service in Intelligent Agriculture}

In intelligent agriculture, IOT identification is an important resource [11], which has a same life cycle with resource objects. Through identification, we can not only carry out unified query on resource data, but also save the relationship between objects in identification, thus to be convenient for user to acquire relevant resource data of object by identification. Therefore, it is considered to use name service to solve query problems of relevant resource data in intelligent agriculture.

Scene and Problems of Intelligent Agriculture. According to the actual situation in reality, this paper designs a four-layer system structure of intelligent agriculture, as shown in Fig.1, of which are respectively object sensing layer, data layer, service layer and application layer form bottom to top. The sensing layer is a heterogeneous network composed of various sensing devices, it is responsible 
for acquiring sensing information in the farmland. The data layer is mainly responsible for maintaining sensing data and description information of entity object, providing query interface. The user puts all access to data needs in application layer. The basic service layer plays a vital role in the system. It needs down to organize the query interface of shared resource data, and up to receive the request of application layer, return corresponding resource to the user. Liu Yang et al [12] think that identification has an important attribute of same life cycle with goods, on this basis, he proposed the implementation of the basic service through China Internet of Things Name Service Platform (hereafter referred to as NIOT) to realize the basic service layer, provide resolution service [13] and discovery service [14] for users. The system can effectively deal with the heterogeneous, complex, dispersion and other characteristics of agricultural products information, make identification of any farm system can be located to corresponding information server, thus to achieve the purpose of integrating resource information of the various farms and providing a unified service for users.

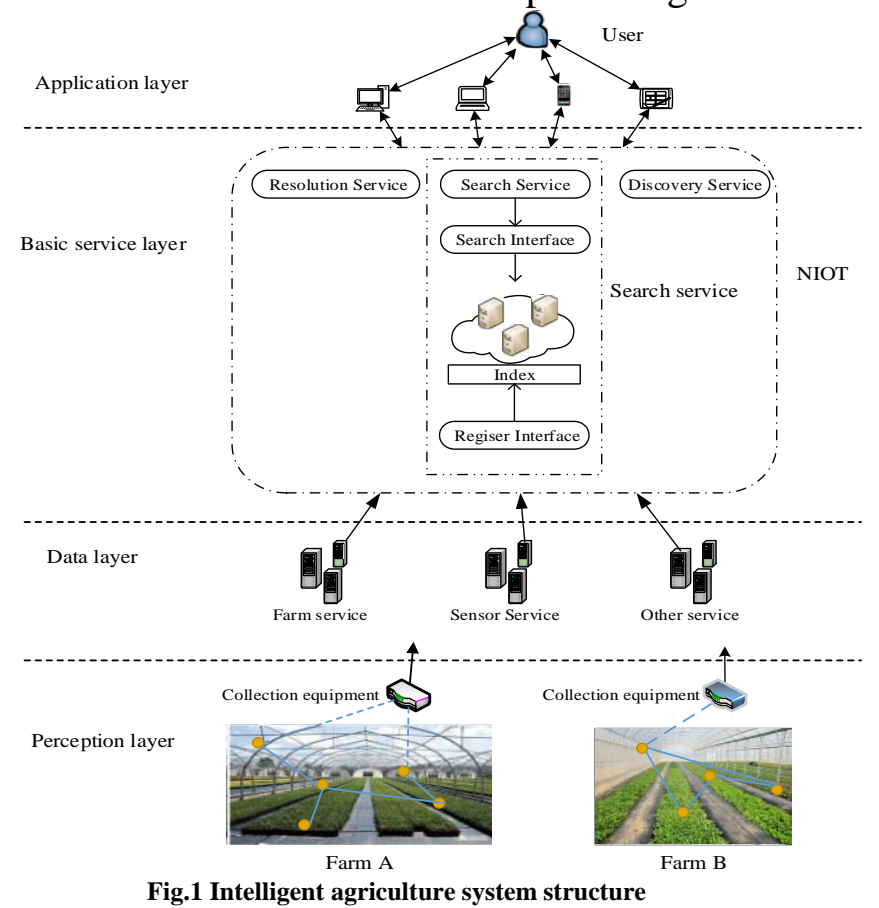

Although NIOT solves the some problems of resource acquisition, it still cannot solve the problem about how to find all data resources related to the product objects according to identification. For example, the temperature sensor deployed in greenhouse records its temperature changes in real time, these temperature data can also be used to describe the vegetable products growing in the greenhouse, but the temperature sensor will not be associated to vegetable products in the greenhouse when it records the temperature data, so the sensing data generated by these sensors cannot be queried through product identification at present. In order to solve this problem, this paper proposes a resource data retrieval method related to objects on the basis of name service.

Basic Definition and Explanation. Definition 1 (Resource Object) Resource object (O) refers to the virtual digital objects extracted from entities in real world. Users can interact with the resource object in network to obtain information of real entities. The property information of resource object (OI) is expressed as an n-tuple of $\left\langle\mathrm{K}_{1}: \mathrm{V}_{1}, \mathrm{~K}_{2}: \mathrm{V}_{2} \ldots \mathrm{K}_{n}: \mathrm{V}_{n}>\right.$, in which $\mathrm{K}_{i}: \mathrm{V}_{i}$ represents the key-value pairs to describe a property of the resource object.

Definition 2 (property) According to the defined resource object, the data that describes the characteristic of object in an aspect is called as the property of this object. Property is generally expressed in the form of <key, value $>$ in resource object, in which key represents the name of description object's feature, value represents the value of this feature. Property can be divided into static property (S-property) and dynamic property (D-property) according to whether it changes with time.

Definition 3 (related objects) For two kind subjects of $\mathrm{O}_{1}$ and $\mathrm{O}_{2}$, if $\mathrm{O} 1$ has the property $\left\langle\mathrm{K}_{i}: \mathrm{V}_{i}>\right.$ that can be used to describe the feature of $\mathrm{O}_{2}$, and any property of $\mathrm{O}_{2}$ cannot describe the feature of 
$\mathrm{O}_{1}$, then $\mathrm{O}_{1}$ is called as the related object of $\mathrm{O}_{2}$. There is a hierarchical relationship between related objects. $\mathrm{O}_{1}$ is the parent object of $\mathrm{O}_{2}$, and $\mathrm{O}_{2}$ is the sub object of $\mathrm{O}_{1}$.

Definition 4 (object identification) According to the hierarchical relationship between objects, assign a unique identification for the resource object or the relationship between them, such an identification is called as object identification $\left(\mathrm{ID}_{O}\right)$, such as " $\mathrm{O}_{1} \cdot \mathrm{O}_{2} \cdot \mathrm{O}_{3} \ldots . \mathrm{O}_{n}$ ". By ensuring the uniqueness of sub object name in parent object, object identification ensures its uniqueness within the whole network.

Definition 5 (relevant information retrieval) Relevant information retrieval of resource object $\left(R_{\text {obj }}\right)$ refers to acquire and query all information resources related to the goal. $R_{\text {obj }}$ not only includes all property information $<\mathrm{K}_{1}: \mathrm{V}_{1}, \mathrm{~K}_{2}: \mathrm{V}_{2} \ldots \mathrm{K}_{n}: \mathrm{V}_{n}>$ of the object itself, but also includes property information $<\mathrm{O}_{1} \cdot \mathrm{K}_{1}: \mathrm{V}_{1}, \mathrm{O}_{2} \cdot \mathrm{K}_{2}: \mathrm{V}_{2} \ldots \mathrm{O}_{n} \cdot \mathrm{K}_{n}: \mathrm{V}_{n}>$ within the related object that can describe its features.

Summary of Retrieval Method on the Basis of Name Service. In intelligent agriculture, the overall flow of retrieval method on the basis of $n$ service is as shown in Fig.2. All farms use a distributed tree structure to maintain the relationship between resource objects, establish a relationship with corresponding resource object with the sensing data as property of object. The farm distributes the object identification to resource object according to its position in the tree structure. The user initiates request according to object identification, the retrieval server obtain the resource object with relevant information retrieval method on the basis of object relationship tree after receiving a request, then returns to users.



Fig 2. Flow chart of retrieval finding service

\section{Resource Relationship Maintenance of the Distributed Tree Structure}

Maintenance of Object and its Association. In IOT, various entities in the real world are abstracted into resource objects to be shared on internet to provide information for people. There are certain hierarchical relationship between these objects, property expressed contents of related objects can be shared. When retrieving relevant resources of object, resource information related to this object should be queried. From the perspective of intelligent agriculture, the number of these associated information with hierarchy is huge and cannot be managed by the search engine with a centralized mode. At the same time, due to the safety need, resource object information should be maintained by farm. Based on above problems, this paper purposes to manage the association between entities objects in IOT with distributed tree structure, the structure is shown in Fig.3.



Fig. 3.Distributed tree structure 
In the tree structure, each object server maintains one or more object nodes. Each object node (ONode) represents a resource object. The object node needs to maintain Record $_{\text {property }}$ and Record $_{\text {subNode }}$ Record property $_{\text {will be introduced in section } 4.2 \text {. The Record }}$ subNode of each node is as shown below.

Record $_{\text {subNode }}=\{$ Name,Info,ExtendProperty $\}$

Among, the Name field represents the name or ID of sub object, which is unique in a same object node. Info field records the position of sub object node in network, which is often saved by the form of IP and interface. If the sub object node is in the server of the object, then it will expressed with "localhost". Extend Property field records the property of the object which can be shared with the sub object, its reservation form is one subset of the object node property key $\left\{\mathrm{key}_{1}, \mathrm{key}_{2}, \ldots, \mathrm{key}_{i}\right\}$.

The distributed tree structure is maintained jointly by each server in the network, each server maintains the property information of managed resource object and the relationship between resource objects. Each object node can be established only by registering in the parent object node it belongs to. This distributed hierarchical tree structure gives the administration of resource object information to the owner of object, each object node can maintain the relationship with all objects in the network by only caring about the relationship with sub node. The object identification of resource object can be generated by its location in the entire tree structure.

Maintenance of Association between Object and Property. A large number of sensors are deployed in various objects of the farm, these sensors periodicity collect the sensing data information which are used to describe some characteristics of the monitoring target with a preset frequency . Various sensing data can be abstracted as the following form:

SensorData $=\{$ RelativeLocation, Property,TimeStamp,Reading $\}$

Among, RelativeLocation can be called the relative address or the described object. This property indicates the relative position of the deployed sensors, as well as can be understood as the resource object monitored by sensor. Property indicates the described property of monitored object. For example, the temperature sensor generally describes the temperature property of monitored object. Video sensor can be expressed as traffic property (monitoring a traffic intersection), security property (monitoring sensitive areas) of monitored object according to demands. TimeStamp represents the time of monitored data acquisition. Reading represents the specific data collected by the sensor. Such as a reading that represents the temperature, a video data stream, a numerical value that represents the light intensity.

According to the four public parts above, we can think these sensing data as the extracted value that describes the property of resource object at a certain time. Therefore, the sensing data generated by the sensor is the dynamic property of resource object. In addition to dynamic property, resource object also has the static property. Content of static property are generally text description information which does not frequently change with the change of time. These property contents are saved in corresponding object nodes in a form of a property record ( Record $_{\text {property }}$ ). Specific content of property record is as following:

Record $_{\text {property }}=\{$ Name,Type,Info $\}$

Among, Name represents the name of property. Type represents the type of property. According to the definition 2, type can be divided into D-property, which is mainly sensor data, and S-property, which is mainly text description. If type is a S-property, text description of the property is saved directly in Info. Different from the S-property, sensing data, as a D-property, is saved in different data servers after being collected by different sensors. Because the object node cannot maintain the massive sensing data, nor can the data server sends all data to the object node again, data can be shared only by opening the query interface. Therefore, if type is a D-property, Info only saves the access interface of sensing data.

In intelligent agriculture, the sensor will not change after completing the deployment of its monitoring object and the property of described object, and all data generated from a sensor is 
maintained and saved by a fixed system. Such a fixed sensing device that monitors the environmental data will not frequently access or leave the network, so this paper registers dynamic property to the farm resource objects by the way of artificial request. When registering, the object identification of objects monitored by sensors, property of representative object and access address of sensor data should be provided. Firstly, the object node monitored by sensor is determined by analyzing the object identification through national platform, then put forward the object property registration request to the object node. This request includes the properties of sensor representative objects and access interface of sensing data, it also claims that the type of property is a dynamic property. In order to obtain data conveniently, we suggest that the data maintenance vendors use the REST service of Web architecture to achieve access interfaces. At the same time, sensor data has a strong time correlation, data of different time points has different meanings, so the access interfaces provided by vendors should support the query request with the condition of time. After successful registration, the access interface of sensor is registered to corresponding object node as the dynamic property that represents the object, thus establishing the relationship between object and sensing data. Static property is generally maintained by the owner of the resource object, when a new static property is added, corresponding property record is added at the object node.

\section{Related Information Retrieval Method Based on Object Relation Tree}

Users have demands to obtain relevant information of retrieval object in IOT resource retrieval. According to the previous conclusion that object identification contains all objects associated with the product, therefore, it is necessary to traverse these objects in retrieval to obtain all information related to the query object. Although the contents of the related object properties can be shared, the sub object only exists in a certain time range of the parent object from the perspective of time. For example, the humidity data in greenhouse can also express the humidity property of vegetable growing in the greenhouse, however, the humidity data recorded by the sensor is the data in the whole life cycle of greenhouse, vegetable products only exist in a certain time range in the greenhouse, so it can only get the sensing data information vegetables in the time range when obtaining relevant data information. Therefore, time range which is associated with products and other related objects must be included when obtaining the sensing data of product related objects. For this purpose, this paper puts forward a resource retrieval method on the basis of identification service, the processing procedure of retrieval server is summarized as follows:

(1) The user obtains the object identification $\left(\mathrm{ID}_{O}\right)$ on physical entity by scanning code and other technical means, put forward query request to the retrieve server of national platform by using the object identification.

(2) After receiving the object identification, the retrieval server firstly analyzes the object identification, locates to the object node (ONode) corresponding to the resource object, obtain the property information (OI) of the resource object in the object node and add to retrieval result set $R_{\text {obj }}$.

(3) This paper defaults to save the duration of query object in related object in the object node as static property. After obtaining all property information of product object, it firstly abstracts the duration as the premise of subsequent query. If the object property does not contain the duration, the query is terminated and return $\mathrm{R}_{\mathrm{obj}}$ to user directly.

(4) In the case that the duration of query object is determined, cyclic query the Record $\mathrm{subNode}_{\text {of }}$ related objects in upper level according to object identification " $\mathrm{O}_{1} \cdot \mathrm{O}_{2} \cdot \mathrm{O}_{3} \ldots . \mathrm{O}_{n}$ ", obtain the Record $_{\text {property }}$ inherited by sub object according to the content of ExtendProperty field, and add

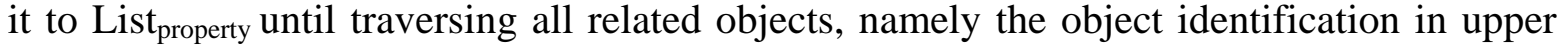
level changes to "cniotroot.cn", then it ends.

(5) Traverse all Record property $_{\text {in List }}$ property, if the Type of the property is S-property, take the Name in record as key, Info as value to add in $\mathrm{R}_{\text {obj. }}$. If the Type of the property is D-property, then take the object duration as condition, send a request to the data access interface corresponding to the Info. Finally, take the return result as value, Name as key to save in $\mathrm{R}_{\mathrm{obj}}$. 
(6) At this time, $\mathrm{R}_{\text {obj }}$ not only includes information $<\mathrm{K}_{1}: \mathrm{V}_{1}, \mathrm{~K}_{2}: \mathrm{V}_{2} \ldots \mathrm{K}_{n}: \mathrm{V}_{n}>$ of query object, but also includes information $<\mathrm{O}_{1} \cdot \mathrm{K}_{1}: \mathrm{V}_{1}, \mathrm{O}_{2} \cdot \mathrm{K}_{i}: \mathrm{V}_{i}, \ldots, \mathrm{O}_{n} \cdot \mathrm{K}_{n}: \mathrm{V}_{n}>$ that can express the object features in the related objects. Finally, return the result set $\mathrm{R}_{\mathrm{obj}}$ to users.

\section{System Implementation}

The retrieval service system designed in this paper applies JAVA to achieve the distributed tree structure maintained by each object server, data server applies data search service with REST style provided by elasticsearch 2.1.1, applies Java language to achieve the resources retrieval service of national platform.

In order to monitor the effectiveness of this system, following test scenario is designed. There are two farms in the scene, they respectively maintain the internal objects and property relationship. A number of different data servers maintain the data generated by sensors for the two farms, one search server is used to receive the user's query request and query relevant data. The CPU of data server and search server are "Intel(R) Core(TM) I3-4160 @ 3.60GHz" with a ROM of 8G. The CPU of farm object server is "Intel(R) Xeon(R) CPU E5-2650 0 @2.00GHz” with a ROM of 16G. Queried agricultural product contains at least 3 related objects.

In performance, the retrieval system of intelligent agriculture mainly concerns the retrieval speed and the influence of great increase of data amount on retrieval time in the network. After testing, the average time consumed is $0.39 \mathrm{~ms}$ for querying among 1 million sensing data by using 4 threads. Fig. 4 is the time consumption change in querying among 1 million sensing data with 6 threads under a condition that the number of sensors increase in intelligent agriculture scene, data server increases and data size expands along.



Fig.4 change of query time along with data size

According to the figure, it can be seen that the overall query time for querying 1 million times with 4 threads is between 39 to 40 seconds when the data size increases from 500 thousand to 20 million. The result shows that the expansion of data size caused by the increase of data service providers does not lead to an exponential increase of query delay. It verifies the method using a distributed tree structure to manage object property relation of IOT has a good expansibility in the heterogeneous network environment of intelligent agriculture.

\section{Conclusions}

Based on consumers' need to acquire all relevant sensing data of products in intelligent agriculture, this paper carries out research on retrieval method of object related sensing resource. It proposes to maintain the relationship between resource objects by using distributed index tree, distributes object identification to actual object, which includes the relation between resources; designs the retrieval scheme that queries all product related resource data by using object identification. Finally, it designs experiment scene, and finds that the retrieval scheme can effectively retrieve the resource data related to object through the test. Under the condition that the number of service providers gradually increases and the data size gradually expand in the network, the retrieval efficiency is basically unchanged, so the system has strong expansibility. At the same time, the scheme also applies to other scenarios, as long as the real objects are in the same logical space, then the sensing data that express 
them at the same time can be shared. Maintaining the relationship between objects through this scheme, data information related to query object can be retrieved by using object identification.

\section{References}

[1] We\&R M. The Computer for the 21st Century.[J]. Scientific American, 1991, 265(1):94-104.

[2] Daoliang Li. Internet of Things and Wisdom Agriculture [J]. Gricultural Engineering , 2012, 02(1):1-7. In Chinese

[3] Tingli Li, Yang Liu, Ye Tian. A Storage Solution for Massive IoT Data Based on NoSQL[M]. The 2012 IEEE International Conference on Internet of Things (iThings), 2012. In Chinese

[4] Yunquan Gao, Xiaoyong Li, Binxing Fang. Survey on the search of Internet of Things [J]. Journal on Communications, 2015, 36(12): 57-76. In Chinese.

[5] Römer K, Ostermaier B, Mattern F, et al. Real-time search for real-world entities: A survey [J]. Proceedings of the IEEE, 2010, 98(11): 1887-1902.

[6] Tingting Sun. Research and Implementation of Semantic Search Engine for Web of Things [D]. Beijing University of Posts and Telecommunications, 2015. In Chinese

[7] Wang H, Tan C C, Li Q. Snoogle: A Search Engine for Pervasive Environments [J]. Parallel \& Distributed Systems IEEE Transactions on, 2010, 21(8):1188-1202.

[8] YAP K K, SRINIVASAN V, MOTANI M. MAX: Human-centric search of the physical world [A]. Proc 3rd Conf Embedded Netw SensorSyst[C].2005.166-179.

[9] Guinard D. A Web of things application architecture[J]. Diss Dgenössische Technische Hochschule Eth Zürich Nr, 2011.

[10] Ostermaier B, RöMer K, Mattern F, et al. A real-time search engine for the Web of Things[C]// Internet of Things (IOT), 2010. IEEE, 2010:1-8.

[11] Ma W, Wu D, Xu D. Uniform Identiifcation System Construction for Agricultural IOT [J]. China Standardization, 2014(1).

[12] Yang Liu. Research on the Key Technologies of Resource Management in the Internet of Things based on Name Service [D]. University of Chinese Academy of Sciences, 2014. In Chinese

[13]Tian Y, Liu Y, Yan Z, et al. RNS-A Public Resource Name Service Platform for the Internet of Things[C]. Green Computing and Communications (GreenCom), 2012 IEEE International Conference on. IEEE, 2012:234-239.

[14]Peng Liu. Research on the Key Technologies of Discovery Service for RFID Network [D]. University of Chinese Academy of Sciences, 2015. In Chinese. 